Hellweg, Joanna

\title{
Fachspezifische Lernbegleitung durch Peer-Learning im Lernzentrum Ernährung, Konsum und Gesundheit der Universität Paderborn
}

Haushalt in Bildung \& Forschung 4 (2015) 4, S. 63-77

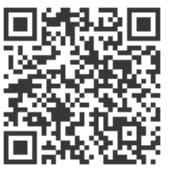

Quellenangabe/ Reference:

Hellweg, Joanna: Fachspezifische Lernbegleitung durch Peer-Learning im Lernzentrum Ernährung, Konsum und Gesundheit der Universität Paderborn - In: Haushalt in Bildung \& Forschung 4 (2015) 4 , S. 63-77 - URN: urn:nbn:de:0111-pedocs-203879 - DOI: 10.25656/01:20387

https://nbn-resolving.org/urn:nbn:de:0111-pedocs-203879

https://doi.org/10.25656/01:20387

in Kooperation mit / in cooperation with:

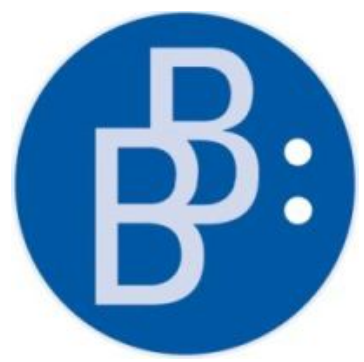

https://www.budrich.de

\section{Nutzungsbedingungen}

Gewährt wird ein nicht exklusives, nicht übertragbares, persönliches und beschränktes Recht auf Nutzung dieses Dokuments. Dieses Dokument ist ausschließlich für den persönlichen, nicht-kommerziellen Gebrauch bestimmt. Die Nutzung stellt keine Übertragung des Eigentumsrechts an diesem Dokument dar und gilt vorbehaltlich der folgenden Einschränkungen: Auf sämtlichen Kopien dieses Dokuments müssen alle Urheberrechtshinweise und sonstigen Hinweise auf gesetzlichen Schutz beibehalten werden. Sie dürfen dieses Dokument nicht in irgendeiner Weise abändern, noch dürfen Sie dieses Dokument für öffentliche oder kommerzielle Zwecke vervielfältigen, öffentlich ausstellen, aufführen, vertreiben oder anderweitig nutzen.

Mit der Verwendung dieses Dokuments erkennen Sie die Nutzungsbedingungen an.

\section{Terms of use}

We grant a non-exclusive, non-transferable, individual and limited right to using this document.

This document is solely intended for your personal, non-commercial use. Use of this document does not include any transfer of property rights and it is conditional to the following limitations: All of the copies of this documents mus retain all copyright information and other information regarding legal protection. You are not allowed to alter this document in any way, to copy it for public or commercial purposes, to exhibit the document in public, to perform, distribute or otherwise use the document in public.

By using this particular document, you accept the above-stated conditions of use.

\section{Kontakt / Contact:}

\section{peDOCS}

DIPF | Leibniz-Institut für Bildungsforschung und Bildungsinformation Informationszentrum (IZ) Bildung

E-Mail: pedocs@dipf.de

Internet: www.pedocs.de

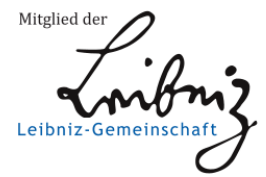




\section{Bildung} Forschung

\section{Aktuelle Fragen der Fachdidaktik Ernährungs- und Verbraucherbildung}

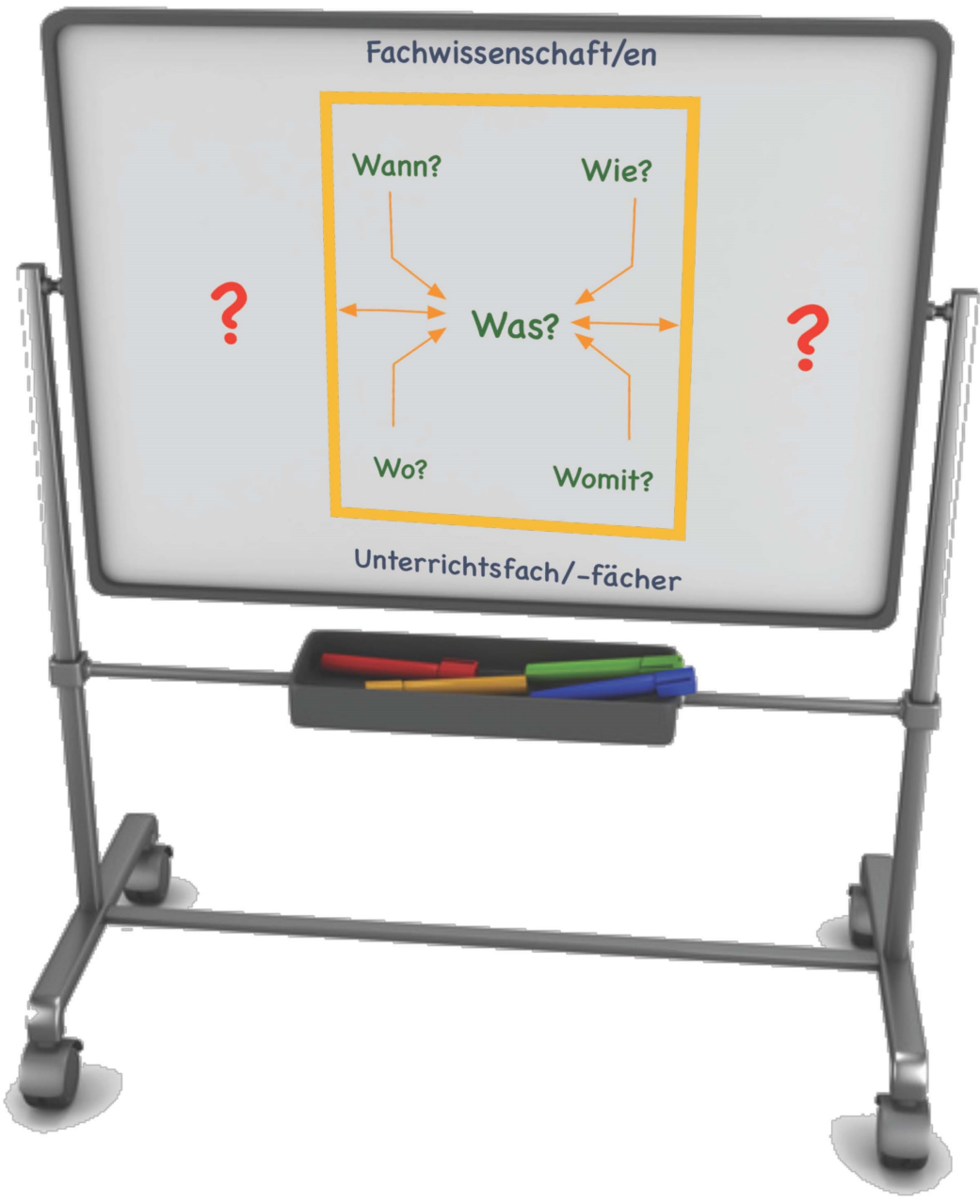


Inhaltsverzeichnis |

Kirsten Schlegel-Matthies \& Claudia Wespi

Editorial. 2

Werner Brandl

Argumente zu Profil und Profilierung einer transdisziplinären Konzeption der Fachdidaktik Ernährungs- und Verbraucherbildung.

Claudia Wespi, Herbert Luthiger \& Markus Wilhelm

Mit Aufgabensets Kompetenzaufbau und Kompetenzförderung ermöglichen.

Veronika Breucker

Empirische Untersuchung zum Einsatz von Bildrezepten als Strukturierungshilfe bei der Zubereitung komplexer Gerichte. Ein Beitrag zur Förderung der Selbstständigkeit von Schülerinnen und Schülern mit einer geistigen Behinderung...

Joanna Hellweg

Fachspezifische Lernbegleitung durch Peer-Learning im Lernzentrum

Ernährung, Konsum und Gesundheit der Universität Paderborn.

Silke Bartsch

Subjektive Theorien von Studierenden zur Nachhaltigen Ernährung.

Explorationsstudie. 78

HaBiFo-Fachtagung 2016 Münster Vielfalt (er)leben - Vielfalt gestalten Konsum, Ernährung und Hauswirtschaft.

Call for Papers Haushalt in Bildung \& Forschung Heft 3/2016

Die ,postmodernen' Konsument/inn/en. 94 
Joanna Hellweg

\section{Fachspezifische Lernbegleitung durch Peer-Learning im Lernzentrum Ernährung, Konsum und Gesundheit der Universität Paderborn}

Der folgende Beitrag stellt das Konzept und die Ziele der fachspezifischen Lernbegleitung im Lernzentrum Ernährung, Konsum und Gesundheit (LEKG) vor, das im Rahmen des Programms „Heterogenität als Chance“ an der Universität Paderborn eingerichtet worden ist, zeigt Herausforderungen und Grenzen aus der Praxis auf und diskutiert weitere Perspektiven für das Studienfach Hauswirtschaft im Institut für Ernährung, Konsum und Gesundheit.

Schlüsselwörter: Lernbegleitung, Peer-Learning, Tutorenausbildung

\section{Hintergründe}

Die Hochschulen in Deutschland sind von der wachsenden Diversität der Studierenden in mehrfacher Hinsicht betroffen. Zehn Prozent der Studierenden an deutschen Hochschulen sind Bildungsausländer und weitere acht Prozent verfügen über eine Migrationsbiographie (Statistisches Bundesamt, 2015).

Die unterschiedlichen Biographien, Schulerfahrungen, Kenntnisstände, Studienmotive und Erwartungen der heterogenen Studierendenschaft stellen alle Fachbereiche vor die Herausforderung, wie dieser Heterogenität in eigenen Fachkulturen begegnet werden kann, damit möglichst alle Studierenden die gleiche Chance der Qualifizierung und persönlichen Entfaltung durch Bildung erhalten (Leicht-Scholten, 2011, S. 47).

Im Studienfach Hauswirtschaft (Konsum, Ernährung, Gesundheit) fällt die hohe Heterogenität der Studierenden auf, die sich u. a. in divergenten Vorkenntnissen, sprachlichen und kulturellen Unterschieden sowie disparaten Vorstellungen vom Fachstudium manifestiert. ${ }^{1}$

Um dieser heterogenen Studierendenschaft gerecht zu werden, wurden im LEKG Maßnahmen erarbeitet, die eine individuelle Betrachtung der Studierenden ermöglichen, unterschiedliche Unterstützungsbedarfe und Disparitäten der Lernenden wahrnehmen und durch zielgruppengerechte Angebote aufarbeiten.

Das Lernzentrum EKG, das im Wintersemester 2011/2012 für Lehramtsstudierende des Studienfachs Hauswirtschaft entstanden ist, intendiert die Entwicklung, Erhebung, Organisation, Koordination und Evaluation von Maßnahmen zur Förderung der Qualität der Lehre und Studienbedingungen. Durch die Bereitstellung von 


\section{Fachspezifische Lernbegleitung}

zusätzlichen, fachintern erarbeiteten Unterstützungsmaßnahmen soll der kontinuierliche Aufbau von fachlichen und fachübergreifenden Kompetenzen bei allen Studierenden bestmöglich unterstützt werden. Eine zielgruppengerechte, fachspezifische Begleitung und Förderung in allen Phasen des Student-Life-Cycle soll gewährleistet werden. Die Entwicklung von Maßnahmen zur Förderung des selbstgesteuerten und kooperativen Lernens für einen fachübergreifenden Kompetenzaufbau und eine grundlegende Studierfähigkeit sowie die Förderung von Problemlösekompetenzen gehören dabei zu den übergeordneten Zielen. Durch den integrativen Arbeitsansatz des LEKG werden Vielfalt und Heterogenität bewusst gefördert und auch als Bereicherung anerkannt.

\section{Das Konzept der fachspezifischen Lernbegleitung}

Die fachspezifische Lernbegleitung im LEKG ist eine auf das Fach Hauswirtschaft (Konsum, Ernährung, Gesundheit) ausgerichtete Lernbegleitung von Studierenden durch Studierende. Sie basiert auf einem systemisch-konstruktivistischen, ressourcenorientierten Ansatz und stellt Stärken sowie Potenziale der Lernenden in den Vordergrund des Lernbegleitungsprozesses (Nestmann, Engel \& Sickendiek, 2014, S. 731). Es handelt sich dabei um eine Interaktion auf Augenhöhe, allerdings mit unterschiedlich verteilten Kompetenzen und Ressourcen, in der fachintern ausgebildete Tutorinnen und Tutoren Studierende bei fachspezifischen Fragen und Anliegen unterstützen und begleiten. Die Unterstützung äußert sich in der gemeinsamen Suche nach förderlichen bzw. hinderlichen Merkmalen des Lernens im Sinne von metakognitiver Reflexion, um im nächsten Schritt geeignete Strategien zu wählen, mit deren Hilfe fachspezifische Anforderungen bewältigt werden können (Weber, 2007, S. 46). In kommunikativen Austauschsituationen sollen die persönlichen Ressourcen und Kompetenzen der Ratsuchenden gestärkt und aktiv in die Bearbeitung ihres Anliegens mit einbezogen werden. Die Studierenden haben die Möglichkeit, ihre bisherigen Lernvorgehensweisen zu reflektieren, notwendige Ressourcen für den Veränderungsprozess zu mobilisieren und individuell passende Handlungswege zu erarbeiten (Schiersmann \& Weber, 2013, S. 211). Ziel ist es, studentische Selbstlern- und Problemlösekompetenzen zu fördern, damit fachliche Anforderungen im gesamten Studienverlauf erfolgreich bewältigt werden können.

In unterschiedlichen Workshops, Diskussionszirkeln und individuellen Lernbegleitungsgesprächen erfahren Studierende u. a. Hilfestellung beim Verfassen von wissenschaftlichen Arbeiten, studientypischen Präsentationen oder der Vorbereitung auf fachliche Prüfungen. Der Schwerpunkt der Lernbegleitungsangebote liegt im fachdidaktischen Bereich, da im Fach „Konsum, Ernährung, Gesundheit“ zukünftige Lehrkräfte ausgebildet werden und die bestmögliche Förderung berufsbezogener Kompetenzen (sowohl im Fach selbst als auch in außercurricularen Supporteinrichtungen wie dem LEKG) im Vordergrund steht. Im Rahmen der fachdidaktischen 


\section{Fachspezifische Lernbegleitung |}

Lernbegleitung bekommen Studierende Unterstützung und Hilfestellung bei der Erstellung und Konzeption von Unterrichtsentwürfen und Praktikumsberichten, bei Fragen zum fachinternen Curriculum der Ernährungs- und Verbraucherbildung (REVIS: Bildungsziele, Kompetenzen, Inhalte und Themen) und bei der Vorbereitung auf die fachdidaktischen Prüfungen.

Techniken, Methoden und Strategien, welche die Stärkung der Autonomie und Selbstorganisation der Lernenden unterstützen, haben in Lernbegleitungssettings des LEKG die höchste Priorität.

\subsection{Ablauf und Phasen der Lernbegleitung}

Die Lernbegleitungsgespräche zwischen Studierenden und Tutorinnen folgen einem bestimmten Ablauf, der durch verschiedene Phasen gekennzeichnet ist.

$\mathrm{Zu}$ diesen Phasen gehören das Schaffen einer tragfähigen Beziehung und einer emotionalen Sicherheit, die adäquate Klärung des Anliegens und der Erwartungen und Ziele der Ratsuchenden, die Stärkung der inneren Ressourcen und Selbstlernpotenziale der Lernenden, sowie die gemeinsame Erarbeitung von Handlungsmöglichkeiten (Schiersmann \& Weber, 2013, S. 216). In den jeweiligen Phasen werden die unten aufgeführten Fragen mit den Studierenden gemeinsam bearbeitet.

\section{Phasen und Fragen zum Einsatz in Lernbegleitungsgesprächen}

- Klärung des Anliegens

Was ist dein Anliegen? Womit bist du unzufrieden? Warum kommst du nicht weiter? Was hast du bereits unternommen, um das Anliegen zu bearbeiten? Welche Erwartungen hast du an das Gespräch?

- Verstehen der Situation und des Anliegens

Wie ist deine persönliche Sichtweise bezüglich des Anliegens? Was sind mögliche Ursachen und Gründe dafür? Was passiert, wenn sich nichts ändert?

- Konkretisierung der Ziele

Welche Ziele bezüglich deines Anliegens verfolgst du? Wie viel Zeit und Energie möchtest du in die Bearbeitung investieren? Was ist machbar und was ist eher unrealistisch?

- Ressourcenaktivierung

Wie kannst du dein Ziel erreichen? Mit welchen Methoden und Strategien warst du bis jetzt erfolgreich? Was hat bis jetzt gut funktioniert? Wie motiviert bist du zum jetzigen Zeitpunkt? Welche deiner Stärken kannst du für die Bearbeitung deines Anliegens aktiv einsetzen?

- Festlegung der Vorgehensweise

An welcher Stelle benötigst du noch Hilfe (z. B. Lerntechniken, strukturierte Vorgehensweisen, Zeitplanung, etc.)? 


\section{Fachspezifische Lernbegleitung}

Was wirst du bis zum nächsten Mal konkret tun? Wie hoch schätzt du die Wahrscheinlichkeit ein, dass dir dies gelingt?

- Vereinbarung über ein weiteres Lernbegleitungsgespräch

Möchtest du ein weiteres Lernbegleitungsgespräch in Anspruch nehmen? Wann sollte das nächste Gespräch erfolgen? Welche Schritte unternimmst du bis zum nächsten Mal? (Siebert, 2009, S. 101)

Diese Fragen sollen Studierende zum Nachdenken sowie Konkretisieren allgemeiner Aussagen, als auch zum Strukturieren ihres Anliegens anregen. Dabei ist eine schrittweise Vorgehensweise wichtig, die nicht unterschiedliche Phasen miteinander vermischt (Siebert, 2009, S. 100).

\subsection{Anforderungen an die lernbegleitenden Tutorinnen und Tutoren}

Die Rolle der Tutorinnen und Tutoren als fachspezifische Lernbegleiterinnen und begleiter ist mit besonderen An- und Herausforderungen verbunden. Sie benötigen für die Ausübung ihrer Tätigkeit eine Kombination aus Wissen, Können und Haltungen, die sich auch als Feld-/Format-/Verfahrenskompetenz bezeichnen lässt (Jokanovic \& Szczyrba, 2014, S. 105). Wissen über die Sachlogik und die fachlichen Anforderungen, die wissenschaftlichen Vorgehensweisen und die Passung von (Fach)Sprache sind für fachspezifische Beratungs- und Begleitungsformate unabdingbar (Jokanovic \& Szczyrba, 2014, S. 106). Lernbegleitende Tutorinnen und Tutoren benötigen überdies grundlegende psychologische Kenntnisse, beispielweise zu Gehirnfunktionen, Lernen im Erwachsenenalter, Lern- und Motivationstheorien oder metakognitiven Prozessen (Siebert, 2009, S. 94). Diese Kenntnisse sind für die Durchführung von Lernbegleitungsgesprächen von entscheidender Bedeutung, da sie einerseits die Klärung und Analyse von Schwierigkeiten erleichtern, welche den Lernprozess blockieren bzw. negativ beeinflussen und andererseits bei der Auswahl entsprechender Interventionen (Auswahl von Methoden, Lerntechniken etc.) helfen können (Landwehr \& Müller, 2006, S. 62).

Lernprozesse werden auf vielfältige Weise blockiert. Oft entstehen Blockaden u. a. auf der Sachebene, wenn ein notwendiges Vorwissen fehlt und so bestimmte Zusammenhänge nicht bzw. falsch verstanden werden. Blockaden können ebenso auf der Methodenebene entstehen, wenn das erforderliche Know-how bei der Lösung einer Aufgabe fehlt und eine inadäquate Vorgehensweise gewählt wird, die den Lösungsweg unnötig erschwert. Störungen im Lernprozess können aber auch auf der kommunikations- oder personenbezogenen Ebene vorkommen, zu denen u. a. ungelöste Konflikte in privaten Beziehungen, fehlende Arbeitsplanung oder mangelnde Arbeitsdisziplin gehören (Landwehr \& Müller, 2006, S. 63). In Lernbegleitungssettings versuchen Tutorinnen und Tutoren gemeinsam mit den Lernenden diese Blockaden bzw. Schwierigkeiten zu identifizieren und zu beseitigen. Die genaue Klä- 


\section{Fachspezifische Lernbegleitung |}

rung und das Verstehen des Anliegens bilden das Gerüst für den weiteren Verlauf des Gesprächs und ermöglichen eine bessere Passung von Methoden und Vorgehensweisen zur Zielerreichung.

Auf der Methodenebene wird von den Tutorinnen und Tutoren erwartet, dass sie gezielt Übungen wählen und einsetzen können, die den Ausbau von strategischen Selbstlernkompetenzen fördern (Kröpke, 2015 S. 36). Die regelmäßige Einbindung und integrative Vermittlung von lernstrategischem Wissen in Lernbegleitungssituationen, sollte Studierende Schritt für Schritt bei der Bewältigung von fachlichen Anforderungen selbstständiger werden lassen und ihr individuelles Wissen und Handeln bemerkbar verbessern. Das Ausmaß der Förderung hängt im Wesentlichen von der Bereitschaft und dem Willen der Lernenden $a b$, sich auf neue und manchmal ungewohnte Aufgaben einzulassen (Siebert, 2009, S. 60).

\subsection{Qualitätsmerkmale, Kompetenzen und Indikatoren für lernbegleitendes Handeln}

Kompetenzerwartungen und -anforderungen an die Tutorinnen und Tutoren beziehen sich auf die Gestaltung von Interaktionsprozessen mit Lernenden, in denen Motivierung, Aktivierung und Förderung der Lernenden sichtbar werden sollen. Die Tabelle 1 (in Anlehnung an Nationales Forum Beratung in Bildung, Beruf und Beschäftigung (nbf) und Forschungsgruppe Beratungsqualität am Institut für Bildungswissenschaft der Ruprecht-Karls-Universität Heidelberg (Hrsg.), 2011) konkretisiert die prozessbezogenen Kompetenzen von Tutorinnen und Tutoren in Lernbegleitungssituationen, ohne Anspruch auf Vollständigkeit zu erheben.

Tab. 1: Qualitätsmerkmale, Kompetenzen und Indikatoren für lernbegleitendes Handeln

\begin{tabular}{|c|c|}
\hline \multicolumn{2}{|c|}{ Qualitätsmerkmale, Kompetenzen und Indikatoren } \\
\hline Beziehungsgestaltung & $\begin{array}{l}\text { Lernbegleitende sind bereit und in der Lage, einen für } \\
\text { den Begleitungsprozess angemessenen Beziehungsaufbau } \\
\text { zu schaffen und für emotionale Sicherheit zu sorgen, die } \\
\text { eine notwendige Grundlage für die gesamte Lernbegleitung } \\
\text { darstellt. } \\
\text { Die Mitwirkung der Lernenden ist hierfür notwendig. }\end{array}$ \\
\hline
\end{tabular}

Lernbegleitende:

$>$ sorgen für eine angenehme Atmosphäre, in der sich Studierende sicher und ernst genommen fühlen;

$>$ machen ihre Rollen bzw. Funktionen transparent;

$>$ berücksichtigen verbale und nonverbale Kommunikationsebenen. Feedback und Metakommunikation sind Bestandteile ihres Handelns;

$>$ gestalten den Beratungsverlauf, die Kommunikation, die Methodenwahl etc. so, dass sie für die Ratsuchenden verständlich und nachvollziehbar sind. Struktur, Ablauf und Methoden werden gemeinsam besprochen;

$>$ beziehen die Lernenden so weit wie möglich in die Steuerung des Lernbegleitungs- 


\section{Fachspezifische Lernbegleitung}

\begin{tabular}{|c|c|}
\hline \multicolumn{2}{|c|}{$\begin{array}{l}\text { prozesses mit ein. Die Wertschätzung der Ressourcen der ratsuchenden Personen } \\
\text { steht dabei im Vordergrund; } \\
\text { sind authentisch, wertschätzend und emphatisch; } \\
\text { bringen den Lernenden Respekt und Wertschätzung entgegen und erwarten von } \\
\text { diesen ebenfalls eine respektvolle Haltung; } \\
\text { gestalten die angemessene Nähe und Distanz zu den Lernenden situationsadäquat. }\end{array}$} \\
\hline $\begin{array}{l}\text { Klärung des Anliegens } \\
\text { und Vereinbarung über } \\
\text { den weiteren Fortgang } \\
\text { der Lernbegleitung }\end{array}$ & $\begin{array}{l}\text { Lernbegleitende sind bereit und in der Lage, im Dialog } \\
\text { mit den Lernenden eine adäquate Klärung des Anliegens } \\
\text { und der Erwartungen vorzunehmen. Gemeinsam wird eine } \\
\text { Vereinbarung über den weiteren Fortgang der Lernbeglei- } \\
\text { tung getroffen. }\end{array}$ \\
\hline \multicolumn{2}{|c|}{$\begin{array}{l}\text { Lernbegleitende: } \\
\text { besprechen zu Beginn des Gesprächs das Anliegen des/der Lernenden und setzen } \\
\text { diese in Beziehung zu den Angeboten der Lernbegleitung, um eine gemeinsame } \\
\text { Auftrags- und Zielklärung zu erreichen (Vereinbarung, Kontrakt); } \\
>\quad \text { arbeiten gemeinsam die Erwartungen an das Ergebnis der Lernbegleitung heraus; } \\
\text { prüfen kontinuierlich, ob sich die Anliegen und Ziele der Lernenden im Verlauf des } \\
\text { Lernbegleitungsprozesses verändern. }\end{array}$} \\
\hline & \\
\hline \multicolumn{2}{|c|}{$\begin{array}{l}\text { Lernbegleitende: } \\
\text { identifizieren gemeinsam mit den Lernenden die Situation und beeinflussende Fak- } \\
\text { toren. } \\
\text { erheben im Einvernehmen mit den Lernenden deren persönliche Daten, vorhandene } \\
\text { Kompetenzen und Ressourcen und interpretieren diese gemeinsam, da sie wissen, } \\
\text { dass vorhandene Kompetenzen und Ressourcen die Grundlage für das Entwickeln } \\
\text { von Lösungen bilden; } \\
\text { arbeiten gemeinsam mit den Lernenden die für die Anliegen relevanten Ziele heraus } \\
\text { und verdeutlichen mögliche Unvereinbarkeiten oder Widersprüche in Hinblick auf } \\
\text { die Zielsetzung der Lernenden. Gegebenenfalls werden gemeinsam Priorisierungen } \\
\text { in Bezug auf die Ziele der Lernenden erarbeitet. }\end{array}$} \\
\hline & \\
\hline \multicolumn{2}{|c|}{$\begin{array}{l}\text { Lernbegleitende: } \\
\quad \text { besprechen gemeinsam mit den Lernenden mögliche Vorgehensweisen; } \\
\text { suchen gemeinsam mit den Lernenden nach Wegen, wie mögliche Hindernisse } \\
\text { überwunden werden können; } \\
\quad \text { vermitteln den Lernenden bedarfs-, personen- und zielgruppengerechte Informatio- }\end{array}$} \\
\hline
\end{tabular}




\section{Fachspezifische Lernbegleitung |}

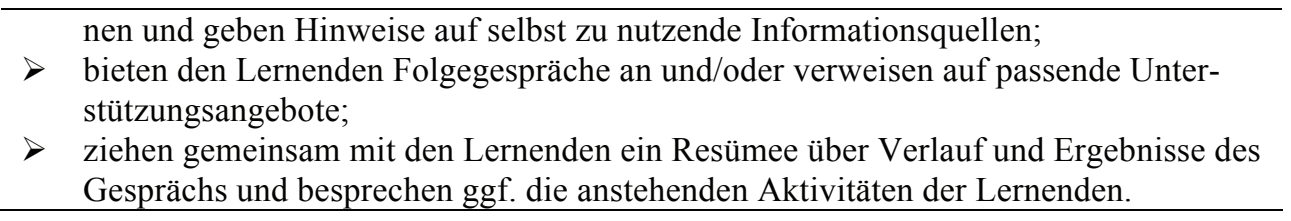

Um die Tutorinnen und Tutoren auf ihre Rolle und die damit zusammenhängenden Aufgaben bestmöglich vorzubereiten, sind entsprechende Qualifizierungsmaßnahmen erforderlich, die sich im Tutorenausbildungsprogramm wiederfinden (Kröpke, 2015, S. 25).

\subsection{Strukturelle Gestaltung des Tutorenausbildungsprogramms}

Die Begleitung von Studierenden und ihrer Lernprozesse ist eine anspruchsvolle Tätigkeit, die eine grundlegende Qualifizierung und kontinuierliche Weiterbildung der Tutorinnen und Tutoren voraussetzt (Kröpke, 2015; Weber \& Katsarov, 2013; Westphal, Stroot, Lerche \& Wiethoff, 2014; Wildt, Szczyrba \& Wildt, 2006).

Das fachinterne Tutorenausbildungsprogramm orientiert sich an den Qualitätsmerkmalen des Nationalen Forums Beratung in Bildung, Beruf und Beschäftigung (nfb) (Schiersmann \& Weber, 2013) sowie an den gemeinsamen Standards der Tutorinnen- und Tutorenausbildung des Netzwerks Tutorenarbeit (Netzwerk Tutorenarbeit an Hochschulen, unveröffentlichtes Manuskript).

Während der gesamten Ausbildung werden die zukünftigen Tutorinnen und Tutoren systematisch an ihre Rolle als Unterstützende und Begleitende von Lernprozessen und auf die damit verbundenen Herausforderungen vorbereitet. Die Ausbildung wird von der Leiterin des LEKG sowie Lehrenden der Fachdidaktik gemeinsam durchgeführt. Die Tutorinnen und Tutoren werden über die gesamte Zeit ihrer tutoriellen Tätigkeit fachintern begleitet und betreut (u. a. durch regelmäßige Teamsitzungen, kollegiale Fallberatung und Supervision). Als Hauptverantwortliche sind Fachdidaktiklehrende zentrale Ansprechpartnerinnen bei der fachdidaktischen Ausrichtung der Lernbegleitung im LEKG. Transparente Vorgaben und Richtlinien sowie genaue Absprachen mit den Tutorinnen und Tutoren in Bezug auf die Zielsetzungen und Erwartungen sind hierfür erforderlich und ermöglichen eine bessere Passung der fachdidaktischen Lernbegleitungsangebote an Unterstützungsbedarfe der Studierenden.

Die Ausbildung erfolgt semesterbegleitend und beinhaltet theoretische Grundlagen, die als Workshopreihen (64 $\mathrm{AE}(\mathrm{AE}=45 \mathrm{~min})$ ) angeboten werden, inhaltlich aufeinander aufbauen und mit Praxis- und Reflexionsanteilen $(90-180 \mathrm{AE})$ verzahnt sind. Durch den hohen Anteil an Praxisphasen zwischen den einzelnen Workshops können die theoretischen Grundlagen mit direkten Erfahrungen aus der Praxis verknüpft werden. 


\section{Fachspezifische Lernbegleitung}

\subsection{Inhaltliche Gestaltung des Tutorenausbildungsprogramms}

Die inhaltlichen Schwerpunkte der Ausbildung setzen sich mit den Grundlagen konstruktivistisch-systemischer Lernbegleitungsansätze, dem Rollenverständnis als Lernbegleiter bzw. Lernbegleiterin, den Qualitätsmerkmalen und Indikatoren für Lernbegleitungsgespräche sowie der methodischen und didaktischen Gestaltung von Lernbegleitungssituationen auseinander. Darüber hinaus folgen die Einführungen in die fachdidaktische Lernbegleitung sowie Grundlagen wissenschaftlichen Arbeitens. Die Reflexion der eigenen Tätigkeit als Lernbegleiter bzw. Lernbegleiterin wird in Kurz-Simulationen und in der Kollegialen Fallberatung angeleitet und ermöglicht.

Die Schulung findet in Kleingruppen mit maximal fünf Personen statt, um eine intensive und auf individuelle Bedürfnisse angepasste Arbeit zu ermöglichen. Nach der Klärung des Rollenverständnisses und den damit zusammenhängenden Kompetenzanforderungen, werden die Teilnehmenden in die Abläufe der Lernbegleitungsprozesse herangeführt, bei denen die Erkenntnisse aus systemischkonstruktivistischen und ressourcenorientierten Lernbegleitungsansätzen eine zentrale Rolle spielen (Siebert, 2009, S. 43). Anhand von Aufgaben zur Selbstreflexion befassen sich die Teilnehmenden mit subjektiven Wissenskonstruktionen und heterogenen Lernpräferenzen (Lernstile, Arbeitstechniken und Methoden, Lernverhaltensweisen) und diskutieren diese im Hinblick auf ihre Relevanz für Lernbegleitungsprozesse (Arnold \& Siebert, 2006; Siebert, 2009, Stelzer-Rothe, 2008; Spitzer, 2002).

Im Workshop zum wissenschaftlichen Arbeiten probieren die Tutorinnen und Tutoren an mehreren Stationen unterschiedliche Schreibübungen zu Themenfindung, Entwicklung von Fragestellungen und Gliederung, Erfassung von Argumentationsstrukturen und Überarbeitungsmethoden wissenschaftlicher Texte aus. Dabei gehen die Übungen auf heterogene Schreibtypen und -stile sowie Schreibschwierigkeiten ein, um den Tutorinnen und Tutoren ihre eigene Diversität bewusst zu machen und diese im gemeinsamen Austausch zu reflektieren. Während der Ausbildung versetzen sich die Teilnehmenden anhand von Kurz-Simulationen in typische Lernbegleitungssituationen und nehmen abwechselnd die Rollen der Lernbegleitenden und Studierenden ein. An konkreten Beispielen werden Gesprächsführungstechniken eingeübt und auf der Metaebene reflektiert. Dabei wird auf die Einhaltung von Feedbackregeln geachtet. Durch das Probehandeln in Experimentalsituationen werden Selbst- und Fremdwahrnehmung geschult und reflektiert (Trautmann \& Sacher, 2010, S. 195).

Die fachdidaktische Lernbegleitung fokussiert vor allem Methoden und Strategien zur Planung und Konzeption von Unterrichtsbausteinen sowie Begleitung der Studierenden bei der Vorbereitung auf fachdidaktische Prüfungen. Dabei geht es u. a. um Fragen zur Themenfindung für Unterrichtsreihen, strukturelle Hilfen beim 


\section{Fachspezifische Lernbegleitung |}

Aufbau von Unterrichtsbausteinen, Formulierung von Lernzielen, Überprüfung der Methodenauswahl in Bezug zu den gesetzten Zielen, als auch um die Auseinandersetzung mit fachdidaktischer Literatur. Dies setzt vor allem das Verständnis von fachdidaktischen Konzepten, Theorien und Methoden sowie die grundlegenden Kenntnisse des Curriculums der Ernährungs- und Verbraucherbildung (REVIS), vor allem in Bezug auf die Bildungsziele und Kompetenzen voraus, da diese wichtige Leitlinien für die Berufspraxis der zukünftigen Lehrerinnen und Lehrer darstellen.

Das nach einem klar strukturierten Schema ablaufende Verfahren der Kollegialen Beratung schließt die Workshopreihen ab. Da in diesem Verfahren an konkreten Fallbeispielen aus der Praxis gearbeitet und vor allem die Synergie der Gruppe genutzt wird, eignet es sich besonders gut für den Einsatz in Gruppen- und Arbeitsteams und kann für die Lösungen berufsspezifischer Problematiken (Konflikt- und Belastungssituationen) beispielsweise bei Lehrkräften genutzt werden (Fallner \& Gräßlin, 1990).

Die schriftliche Abschlussevaluation der Tutorinnen und Tutoren zur Gestaltung des gesamten Tutorenausbildungsprogramms und zum persönlichen Kompetenzzuwachs sowie die Aushändigung der hochschulweit anerkannten Zertifikate (T-Cert) runden das gesamte Ausbildungsprogramm ab.

\subsection{Herausforderungen und Grenzen der Lernbegleitung}

Die Rolle der Tutorinnen und Tutoren ist mit besonderen Herausforderungen verbunden. Als Studierende und gleichzeitige Lernbegleitende sind sie mit doppelten Anforderungen konfrontiert. Auf der einen Seite werden die Tutorinnen und Tutoren stets für ihre Leistungen im Studium, und auf der anderen Seite für ihre Tätigkeit als Peer-Begleitende bewertet. Dieser hohe Leistungsdruck wird besonders in den Abschlussreflexionen des Tutorenausbildungsprogramms thematisiert. Besonders belastend empfinden die Tutorinnen und Tutoren den Zeitraum der Praxisphase, die sie als Studierende im Masterstudium absolvieren müssen. Sie betonen vor allem die schwierige zeitliche Vereinbarung ihrer Tätigkeit im LEKG mit den Anforderungen aus Schule und Studium. In dieser Phase werden sie oft selbst mit Fragen und Unsicherheiten konfrontiert und brauchen dafür kompetente Ansprechpartnerinnen und partner aus der Schulpraxis, die sie als sachkundige Mentorinnen bzw. -mentoren unterstützen und begleiten können.

Die besonderen Herausforderungen an Lernbegleitende ergeben sich auch zum Teil aus einer falsch verstandenen Rolle der Tutorinnen und Tutoren, die von Studierenden als Expertinnen und Experten für fachliche Fragen und fachdidaktische Inhalte und weniger als Begleitende auf der Suche nach Ideen und möglichen Handlungswegen angesehen werden (Henkel \& Vollmer, 2014, S. 55). Die häufigsten Fragen an die Tutorinnen und Tutoren beziehen sich zum Beispiel auf das Verständnis der REVIS-Bildungsziele, die einigen Studierenden erhebliche Interpretations- 


\section{Fachspezifische Lernbegleitung}

schwierigkeiten bereiten sowie die Einordnung selbst geplanter Unterrichtsvorhaben in den Didaktischen Würfel oder die Formulierung der entsprechenden Lehr- und Lernziele. Darüber hinaus führen oft Unsicherheiten der Studierenden bezüglich der Legitimation ihrer Unterrichtsvorhaben oder Unterrichtsbausteine zu einer hohen Anspruchshaltung gegenüber den Lernbegleitenden. Sie erwarten hierfür konkrete Instruktionen bzw. Lösungsvorschläge, die weit über den konstruktiven Austausch hinausgehen. Die Aufklärung über die Funktion der prozessorientierten Lernbegleitung führt bei einigen Studierenden zu spürbaren Enttäuschungen und dem Wunsch nach mehr „professionellen Beratern“, die auch konkrete, inhaltliche Antworten und Lösungen anbieten. Auf der anderen Seite heben jedoch viele Studierende eben diese Beziehung auf Augenhöhe positiv hervor. Sie schätzen das Vermögen der Tutorinnen und Tutoren, sich in die Problemlagen einfühlen zu können und das Wissen um die fachlichen Herausforderungen bei der Bewältigung von Studienaufgaben, die sie aus ihrer eigenen Perspektive als Studierende kennen (Henkel \& Vollmer, 2014, S. $55)$.

Schwierig gestaltet sich aber auch die kurze Dauer der Tätigkeitsausübung als Lernbegleitende. An dem Tutorenausbildungsprogramm des LEKG dürfen ausschließlich Studierende teilnehmen, die alle erforderlichen fachdidaktischen Lehrveranstaltungen abgeschlossen haben, was faktisch erst im vierten Fachsemester des Bachelorstudiengangs erfolgt. Daher müssen direkt zwei Semester später neue Tutorinnen und Tutoren angeworben und ausgebildet werden, um die Kontinuität der Lernbegleitung gewährleisten zu können. Dieser ständige Personalwechsel führt unvermeidlich zu hohen Kompetenzschwankungen innerhalb des Lernzentrums.

Zum Ende ihres eigenen Masterstudiums verfügen die Tutorinnen und Tutoren über einen erheblichen Wissens- und Erfahrungsvorsprung und erfüllen so die Voraussetzungen, um Studierende vor allem beim Übergang vom Bachelor in den Master optimal begleiten und beraten zu können.

\section{3 Überlegungen und Perspektiven}

Das studiengangsbezogene LEKG hat sich zu einem festen Bestandteil der universitären Ausbildung im Fach Hauswirtschaft entwickelt. Dies zeigen die im Wintersemester 2014/2015 erhobenen Daten zur Nutzung des Lernzentrums.

Die Beratungs- und Lernbegleitungsangebote werden seit 2011 kontinuierlich stärker nachgefragt und entsprechend ausgeweitet. Eine Befragung von 132 Studierenden vom zweiten bis zum siebten Fachsemester (B. Ed., M. Ed. und LPO 2003) zeigt, dass 41 Prozent der Befragten die Lernbegleitungsangebote regelmäßig nutzen und gut 62 Prozent auf das Angebot durch Weiterempfehlung von Mitstudierenden aufmerksam wurden. Insbesondere die Lernbegleitungskompetenz der Tutorinnen wird von ca. 77 Prozent der Bachelor-Studierenden (60 Prozent gut / 16 Prozent sehr gut), von ca. 72 Prozent der Master-Studierenden (61 Prozent gut / 11 Prozent sehr 


\section{Fachspezifische Lernbegleitung |}

gut) und von ca. 70 Prozent der Studierenden nach LPO (43 Prozent gut / 26 Prozent sehr gut) als gut bzw. sehr gut bewertet. Auch die Rückmeldungen nach den mündlichen Staatsexamensprüfungen in der Fachdidaktik zeigen ein ähnliches Bild.

Das Ausbildungsprogramm für die studentischen Tutorinnen und Tutoren kann deshalb als erfolgreich bewertet und soll unbedingt weitergeführt werden. Zum einen profitieren die Studierenden vom Lernbegleitungsangebot, zum anderen erwerben aber auch die Tutorinnen und Tutoren professionelle Kompetenzen für den Lehrberuf. $^{2}$

Ein Blick auf die Gründe, die zum Besuch des Lernzentrums führen, zeigt, dass insbesondere im Bereich der Fachdidaktik im Studienverlauf bei den Studierenden Unterstützungsbedarf besteht. 55 Prozent der befragten Studierenden haben im WS 2014/15 Beratungen zum Erstellen eines Unterrichtsentwurfs in Anspruch genommen, außerdem diverse Workshopangebote zur Fachdidaktik sowie weitere Veranstaltungen und Übungen, die von den Studierenden angefragt und daraufhin vom LEKG angeboten wurden. Hier besteht zukünftig weiterhin Bedarf sowohl im Bachelor-, als auch im Masterstudiengang vor allem mit Blick auf das Praxissemester. Der Übergang in das Praxissemester wird zwar curricular durch entsprechende Veranstaltungen vorbereitet, dennoch fehlen hier gerade für die Tutorinnen und Tutoren, die in das Praxissemester gehen, entsprechende Ansprechpartnerinnen und -partner aus der Schulpraxis. Dieses Problem könnte durch eine Kooperation mit Lehramtsanwärterinnen und -anwärtern gelöst werden, die als zusätzliche Beratungs- und Lernbegleitungskräfte im LEKG zur Verfügung stehen würden. Durch ihre unmittelbare Nähe zum schulischen Unterricht und zur gelebten „Praxis vor Ort“ könnten sie die fachliche Ausbildung zukünftiger Lehrkräfte didaktisch gut unterstützen, wie in der Abbildung 1 verdeutlicht wird.

Im Sommersemester 2014 wurde eine weitere Befragung von 102 Studierenden im Bachelorstudiengang zu den Studienbedingungen und zur Lehrqualität durchgeführt. Ersichtlich wurde, dass knapp ein Drittel (32 Prozent) der Studierenden sich bereits während des Semesters mehr und spezifische Rückmeldungen zu ihren Lernfortschritten wünschen, hier gibt es noch Verbesserungsbedarf. In dieser Befragung hielten 91 Prozent der Studierenden eine starke Förderung im Bereich des Berufs- bzw. Praxisbezugs für wichtig, nur 34 Prozent sahen diesen Bezug entsprechend stark gefördert. 80 Prozent hielten die Stärkung von Autonomie und Selbstständigkeit für wichtig. Hier hielten 44 Prozent die Förderung für angemessen. Diese Befunde bilden eine Grundlage für die Entwicklung weiterer Maßnahmen zur Kompetenzanbahnung und -förderung im Lernzentrum EKG (SchlegelMatthies \& Hellweg, unveröffentlichtes Manuskript). 


\section{Fachspezifische Lernbegleitung}

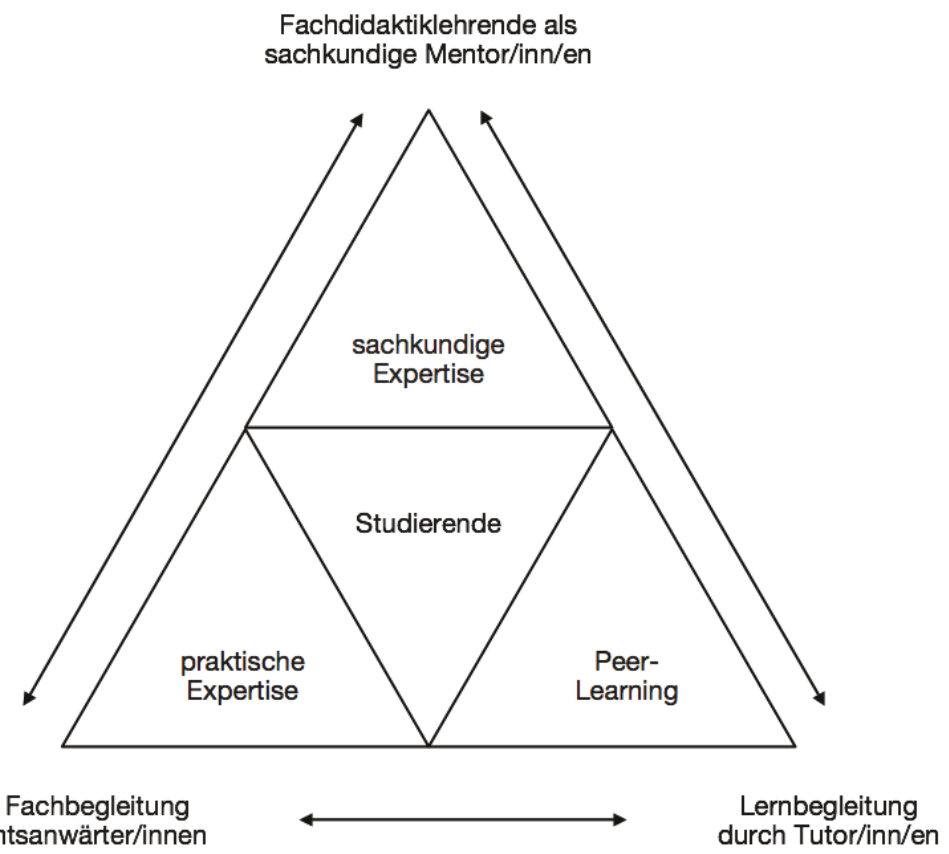

Didaktische Fachbegleitung durch Lehramtsanwärter/innen

durch Tutor/inn/en

Abb. 1: Das didaktische Beratungs- und Lernbegleitungsdreieck des Lernzentrums EKG (Quelle: Eigene Darstellung)

Die neu konzipierten Beratungs- und Lernbegleitungspakete (BLP) für Studierende in unterschiedlichen Fachsemestern sollen hierfür eine zusätzliche Hilfestellung bieten. Es handelt sich dabei um drei Beratungs- und Lernbegleitungsformate, wobei die BLP 1 und 2 mit E-Learning-Angeboten zur Selbstreflexion ergänzt werden:

- BLP 1 „Sicher starten im Fach KEG“ für Studierende des 1.-2. Semesters (B. Ed.)

- BLP 2 „Sicher vorankommen im Fach“ für Studierende des 3.-6. Semesters (B. Ed.)

- BLP 3 „Sicher in die Schulpraxis“ für Studierende des 7.-8. Semesters (M. Ed.)

Die gezielte Unterstützung in drei Abschnitten ist aufgrund der Ergebnisse der kontinuierlich durchgeführten Evaluationen und Beobachtungen LEKG entwickelt worden. So sollen alle Phasen des Student-Life-Cycle berücksichtigt werden, um eine stetige Kompetenzentwicklung gemäß den jeweiligen Anforderungen im Semester zu sichern. Die Konzeption dafür soll im Rahmen einer Zukunftswerkstatt mit Lehrenden, Studierenden sowie Tutorinnen und Tutoren entwickelt werden. Die Beratungs- und Lernbegleitungsangebote sollen so aufeinander aufbauen, dass 


\section{Fachspezifische Lernbegleitung |}

sie sich im gesamten Studienverlauf bestmöglich ergänzen. Die Zukunftswerkstatt dient dem gemeinsamen Erfahrungsaustausch, der Herstellung von Transparenz der Beratungs- und Lernbegleitungsangebote sowie der Einholung des Einverständnisses aller Beteiligten für die geplante Weiterentwicklung des LEKG. Die konzeptionelle Erarbeitung des Angebots richtet sich stark nach der „Charta guter Lehre“ (Jorzik, 2013), die Grundsätze und Leitlinien für eine bessere Lehrkultur umfasst.

Außerdem sollen alle Maßnahmen in regelmäßigen Abständen evaluiert und verbessert werden. Durch die Beteiligung der Studierenden und aller Lehrenden der Fachdidaktik soll die gemeinsame Verantwortung für den Lern- und Studienerfolg strukturell verankert werden.

\section{Anmerkungen}

1 Erhebung zur Entwicklung des Lernzentrums EKG: Auswertung der Umfrageergebnisse mit 100 Studierenden im Fach Hauswirtschaft (Konsum, Ernährung, Gesundheit) im WS 2011/12.

2 Ergebnisse aus den Abschlussreflexionen von Tutorinnen und Tutoren im SoSe 2014.

\section{Literatur}

Arnold, R. \& Siebert, H. (2006). Konstruktivistische Erwachsenenbildung. Von der Deutung zur Konstruktion von Wirklichkeit. Baltmannsweiler: Schneider Verlag Hohengehren.

Fallner, H. \& Gräßlin, H.-M. (1990). Kollegiale Fallberatung. Eine Systematik zur Reflexion des beruflichen Alltags. Hille: Ursel Busch Fachverlag.

Henkel, Ch. \& Vollmer, A. (2014). Beraten auf Augenhöhe: Ein theoretisches Modell der Peer-Beratung. In P. Westphal, T. Stroot, E. Lerche \& Ch. Wiethoff (Hrsg.). (2014), Peer Learning durch Mentoring, Coaching \& Co. Aktuelle Wege in der Ausbildung von Lehrerinnen und Lehrern (S. 51-58). Immenhausen bei Kassel: Prolog-Verlag.

Jokanovic, M. \& Szczyrba, B. (2014). Tutorienarbeit an Hochschulen. Professionalisierung der Lehre „, bottom up “. Neues Handbuch der Hochschullehre (NHHL 20014 03, S. 103-120). Raabe Fachverlag für Wissenschaftsinformationen.

Jorzik, B. (Hrsg.). (2013). Charta guter Lehre. Grundsätze und Leitlinien für eine bessere Lehrkultur. Essen: im Auftrag des Stifterverbandes der Deutschen Wissenschaft.

Kröpke, H. (2015). Tutoren erfolgreich im Einsatz. Ein praxisorientierter Leitfaden für Tutoren und Tutorentrainer. Opladen \& Toronto: Verlag Barbara Budrich. 


\section{Fachspezifische Lernbegleitung}

Landwehr, N. \& Müller, E. (2006). Begleitetes Selbststudium. Didaktische Grundlagen und Umsetzungshilfen. Bern: h.e.p verlag ag.

Leicht-Scholten, C. (2011). Hochschule öffne dich: Wie Vielfalt und Chancengerechtigkeit Hochschulen stärken. In Henrich- Böll-Stiftung (Hrsg.), Öffnung der Hochschule. Chancengerechtigkeit, Diversität, Integration (S. 47-51). Berlin: Heinrich-Böll-Stiftung. Nationales Forum Beratung in Bildung, Beruf und Beschäftigung (nbf) und Forschungsgruppe Beratungsqualität am Institut für Bildungswissenschaft der Ruprecht-Karls-Universität Heidelberg (Hrsg.). (2011). Qualitätsmerkmale guter Beratung. Erste Ergebnisse aus dem Verbundprojekt: Koordinierungsprozess Qualitätssicherung in der Beratung für Bildung, Beruf und Beschäftigung. Bielefeld: Bertelsmann Verlag.

Nestmann, F., Engel, F. \& Sickendiek, U. (Hrsg.). (2014). Das Handbuch der Beratung. Band 2: Ansätze, Methoden und Felder. Tübingen: dgvt-Verlag.

Netzwerk Tutorenarbeit an Hochschulen (2015). Gemeinsame Standards der Tutorinnen- und Tutorenausbildung des Netzwerks Tutorienarbeit. (Unveröffentlichtes Manuskript).

Schiersmann, Ch. \& Weber, P. (Hrsg.). (2013). Beratung in Bildung, Beruf und Beschäftigung. Eckpunkte und Erprobung eines integrierten Qualitätskonzeptes. Bielefeld: Bertelsmann Verlag GmbH \& Co. KG.

Schlegel-Matthies, K. \& Hellweg, J. (2015). Antrag zur 2. Förderlinie des gemeinsamen Programms des Bundes und der Länder für bessere Studienbedingungen und Qualität in der Lehre. (Unveröffentlichtes Manuskript). Paderborn.

Siebert, H. (2009). Grundlagen der Weiterbildung. Selbstgesteuertes Lernen und Lernbegleitung. Konstruktivistische Perspektiven. Augsburg: ZIEL - Zentrum für interdisziplinäres Lernen $\mathrm{GmbH}$.

Spitzer, M. (2002). Lernen. Gehirnforschung und die Schule des Lebens. Heidelberg: Spektrum Akademischer Verlag.

Statistisches Bundesamt (2015). Bildung und Kultur. Studierende an Hochschulen (Vorbericht) (Fachserie 11, Reihe 4.1). Wiesbaden: Statistisches Bundesamt.

Stelzer-Rothe, T. (Hrsg.). (2008). Kompetenzen in der Hochschullehre. Rüstzeug für gutes Lehren und Lernen an Hochschulen. Rinteln: Merkur Verlag.

Trautmann, M. \& Sacher, J. (Hrsg.). (2010). Unterrichtsentwicklung durch Videofeedback. Besser kommunizieren lernen. Göttingen: Vandenhoeck \& Ruprecht $\mathrm{GmbH} \& \mathrm{Co}$. KG.

Weber, A. (2007). Problem-Based Learning. Ein Handbuch für die Ausbildung auf der Sekundarstufe II und der Tertiärstufe. Bern: h.e.p verlag ag.

Weber, P. \& Katsarov, J. (2013). Die Qualitätsmerkmale für die Beratung - Entwicklung und Erprobung. In Ch. Schiermann \& P. Weber, Beratung in Bildung, Beruf und Beschäftigung. Eckpunkte und Erprobung eines integrierten Qualitätskonzeptes. Bielefeld: Bertelsmann Verlag GmbH \& Co. KG. 


\section{Fachspezifische Lernbegleitung |}

Westphal, P., Stroot, T., Lerche, E. \& Wiethoff, Ch. (Hrsg.). (2014). Peer Learning durch Mentoring, Coaching \& Co. Aktuelle Wege in der Ausbildung von Lehrerinnen und Lehrern. Immenhausen bei Kassel: Prolog-Verlag.

Wildt, J., Szczyrba, B. \& Wildt, B. (Hrsg.). (2006). Consulting Coaching Supervision. Eine Einführung in Formate und Verfahren hochschuldidaktischer Beratung. Bielefeld: Bertelsmann Verlag GmbH \& Co. KG.

\section{Verfasserin}

Joanna Hellweg

Universität Paderborn

Institut für Ernährung, Konsum und Gesundheit

Warburger Straße 100

D-33098 Paderborn

E-Mail: joanna.hellweg@uni-paderborn.de 